\title{
Análise do Timi Risk score em pacientes com infarto agudo do miocárdio com supradesenvolvimento do segmento ST após 6 meses* $^{*}$
}

\section{The Timi Risk score analysis in ST-elevation myocardial infarction patients after 6 months}

\author{
Bernardo Mazzini Ketzer(1), Érlon Gil(1), Gustavo Reis \\ Rodrigues (1), Marcelo Fukuhara Kawata(1), Carlos Gun $^{(2)}$, \\ Fábio Augusto de $\mathrm{Luca}^{(3)}$
}

\begin{abstract}
Ketzer BM, Gil E, Rodrigues GR, Kawata MF, Gun C, Luca FA. Análise do TIMI risk score em pacientes com infarto agudo do miocárdio com supradesenvolvimento do segmento ST após 6 meses. Rev Med (São Paulo) 2003 jan.-dez.;82(1-4)58-66.

RESUMO: Introdução: O escore de TIMI RISK (TR) para infarto agudo do miocárdio com supradesnivelamento do segmento ST (IAM) foi descrito originalmente por Morrow e cols. (2000), baseado em 8 variáveis. Foi utilizado no estudo In TIME II como preditor de morte em 30m dias e diversos estudos têm relatado com sucesso o TR como índice prognóstico para IAM, no entanto poucos relatos avaliam a evolução tardia desses pacientes e relação como o escore. Objetivo: Avaliar a evolução em 6 meses dos pacientes internados em Hospital Escola com IAM e sua relação com a estradição do TR. Casuística e Método: Foram acompanhados ambulatorialmente em hospital escola, 89 pacientes, durante 6 meses após determinação inicial do escore do TR realizado durante internação por IAM. Foram avaliados os seguintes parâmetros: óbito, reinternação por evento cardiovascular e necessidade de revascularização. Divididos em 3 grupos de acordo com o escore: Grupo I $(0,1,2)$; Grupo II $(3,4,5)$; Grupo III (> 5), foram submetidos à análise estatística pelo método de Qui-Quadrado. Resultados: Óbito, revascularização e reinternação respectivamente: GI- 4,1\%, 20\%, 25\%; GII- 10\%, 30\% e GIII$52 \%, 48 \%, 60 \%$. Conclusão: O escore de TR manteve significativo aumento de eventos nos grupos II e III em relação ao grupo I; mostrando a possível aplicabilidade do escore também como preditor a longo prazo (6 meses).
\end{abstract}

DESCRITORES: Infarto do miocárdio/mortalidade. Seguimentos. Fatores de risco. Pacientes internados. Revascularização miocárdica. Qui-quadrado. Tempo de internação.

\section{INTRODUÇÃO}

As doenças cardiovasculares representam a principal causa de morbi-mortalidade da atualidade, sendo a doença arterial coronariana (DAC) seu principal expoente devido a alta prevalência e estreita correlação com o estilo de vida contemporâneo ${ }^{1}$.

O desequilíbrio entre a oferta e a demanda de

\footnotetext{
*Vencedor XXI COMU-2002, área Clínica

(1) Acadêmicos do $4^{\circ}$ Ano da Faculdade de Medicina da Universidade de Santo Amaro.

(2) Orientador, Prof. Titular da Disciplina de Cardiologia da Universidade de Santo Amaro.

(3) Orientador, Médico Cardiologista, Coordenador da Residência Médica de Cardiologia da Universidade de Santo Amaro.

Endereço para correspondência: Gustavo Reis Rodrigues. Rua Joaquim Távora, 1020 - Apto. 22B, Vila Mariana, São Paulo, SP, CEP: 04015-012. e-mail: cfr@terra.com.br
} 
oxigênio para o miocárdio caracteriza DAC, cuja principal etiologia é aterosclerose (Figura 1). O processo de instabilização da placa aterosclerótica está relacionada a fatores inflamatórios, podendo causar fissura ou até ruptura hemorrágica da mesma (Figura 2), levando a instalação da síndrome coronariana aguda (SCA), caracterizada pelo infarto agudo do miocárdio (IAM), Angina Instável e Morte Súbita ${ }^{1}$.

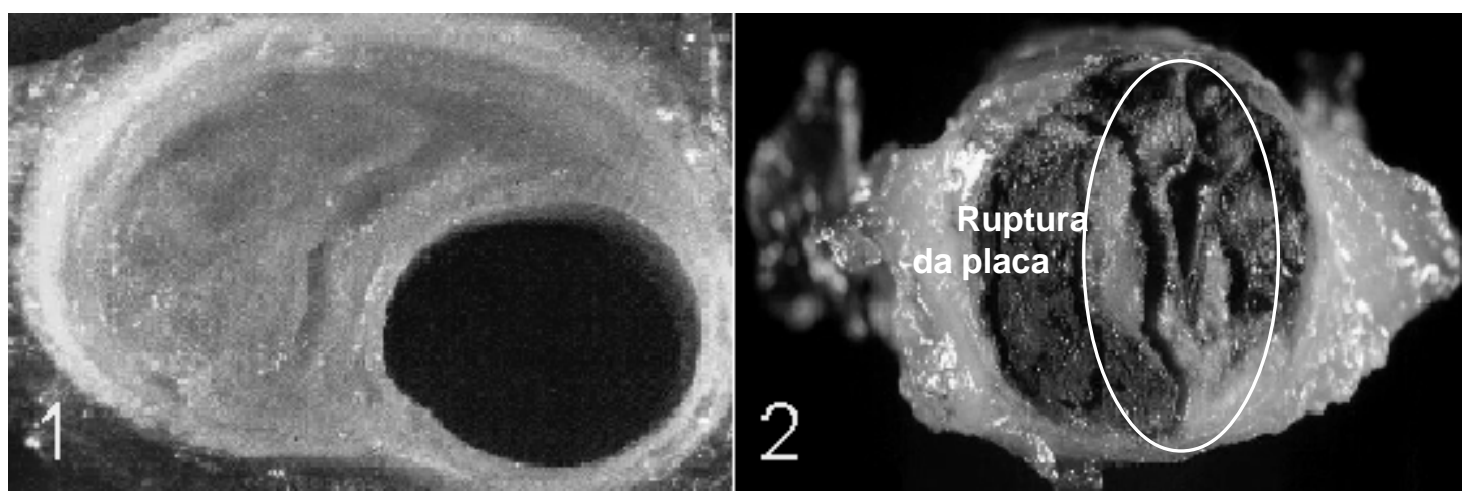

Figura 1. Foto 1 - mostra o comprometimento arterial por placa de ateroma; Foto 2 - corte de artéria mostrando ruptura da placa ateromatosa

Ressaltando a importância da SCA estima-se que a angina instável seja uma das causas mais freqüentes de hospitalização, sendo a maior causa de internação em unidade coronariana. Nos EUA, o número de internações com diagnóstico de angina instável ultrapassa 600.000 por ano ${ }^{15}$. Apesar dos altos custos na prevenção e no tratamento do IAM, este continua sendo a principal causa de óbito na atualidade.

Nos EUA os encargos anuais são aproximadamente de 30 bilhões de dólares. Neste país quase 1,5 milhões de pessoas sofrem anualmente de IAM, cerca de 1 paciente a cada 20 segundos $^{1}$.

No Brasil, apesar de não existirem dados fidedignos, estima-se cerca de 300 a 350 mil casos de IAM por ano ${ }^{15}$.

$\mathrm{O}$ aumento da incidência das doenças cardiovasculares, em especial do IAM, faz necessário o estudo de novos métodos de estratificação de risco dos pacientes, utilizando marcadores prognósticos de fácil aplicabilidade, com a intenção de reduzir reinfarto, arritmias, insuficiência cardíaca e outras complicações, assim como a diminuição dos custos com internações desnecessárias, adequação da terapia ao tratamento, encaminhamento do paciente para unidades hospitalares condizentes com sua estratificação ${ }^{7,9,16}$ e a melhora da qualidade das informações prestadas aos familiares dos pacientes ${ }^{8}$.

O tratamento mais específico voltado para o paciente em decorrência de sua estratificação, resultou em diversos estudos, na diminuição dos índices de morbimortalidade, remodelamento ventricular, redução da fração de ejeção, edema agudo de pulmão, choque cardiogênico e insuficiência cardíaca congestiva dos pacientes.

Desde a década de 60 têm-se demonstrado que a mortalidade de indivíduos pós infartados tem relação com fatores hemodinâmicos, radiográficos, eletrocardiográficos e história da doença, sendo que a maioria destes podem ser colhidos minutos após a admissão no hospital ${ }^{3}$.

Apesar dos diferentes critérios analisados, a proposta de muitos trabalhos é a criação de grupos de risco de mortalidade pós infarto. Entre 1973 e 1974, Moss et al. ${ }^{11}$ analisaram 518 pacientes, classificando-os em grupos de risco, para verificar o índice de mortalidade.

O Multicenter Postinfarction Research Group em 1978 por meio de variáveis relacionadas a características isquêmicas, elétricas e mecânicas dos pacientes, criou uma estratificação de risco para IAM, analisando posteriormente a contribuição individual de cada uma das variáveis para avaliação do risco ${ }^{10}$.

Sanz et al. ${ }^{18}$ estudaram 259 pacientes, durante 34 meses, com o objetivo de fazer um acompanhamento de longo prazo, verificar as variáveis de risco mais importantes e caracterizar os diferentes tipos de pacientes que sobreviveram ao IAM.

Kornowski et al. ${ }^{6}$ estudaram a relação entre reinfarto durante um ano e os dados clínicos referentes ao primeiro infarto, admissão atual, e fatores de risco, examinando 3.695 pacientes em unidade israelense de prevenção ao reinfarto.

Foi desenvolvido um índice de comorbidade por Normand et al. ${ }^{13}$, no qual ajustaram a gravidade de cada uma das 46 morbidades pertencentes ao estudo, com o objetivo de avaliar o risco de mortalidade em um grupo não randomizado. Com os resultados concluiram que de acordo com o tipo e o número de morbidades o risco de óbito aumenta.

Dados clínicos colhidos antes da terapia trombolítica foram utilizados por Hillis ${ }^{3}$ para avaliar 3.339 pacientes com IAM, possibilitando a criação de uma estratificação de risco de óbito em 6 semanas. 
The Canadian Assessment of Miocadial Infarction $(\mathrm{CAMI})^{17}$, avaliou a mortalidade de pacientes após IAM durante a internação e após um ano de alta hospitalar, assim como a recorrência de infarto e a necessidade de revascularização em 3.178 pacientes, acompanhados durante dois anos em nove hospitais canadenses.

O Grupo Italiano Per lo Studio della Sopravvivenza nell'Infarto Miocardico (GISSI - 2 trial) utilizou 10.219 pacientes, analisando o risco de óbito pós infarto em seis meses, sendo um dos primeiros a adequar as avaliações de risco ao advento da era trombolítica ${ }^{19}$.

Para expandir as tentativas do Health Care Financing Administration de qualificar o tratamento para pacientes com IAM nos diversos hospitais dos EUA, Sharon et al. ${ }^{12}$ em 1994, avaliaram 14.581 pacientes infartados em diferentes Estados (Alabama, Connecticut, Iowa e Wisconsin) e criaram um preditor de óbito em 30 dias.

Outro estudo que fez um preditor de óbito em 30 dias foi o Global Utilization of Streptokinase and Tissue Plasminogen Activator for Occluded Coronary Arteries (GUSTO - I), o qual avaliou 41.021 pacientes que utilizaram terapias trombolíticas intravenosas, após episódio de IAM, para criar uma estratificação com utilidade na rotina clínica hospitalar ${ }^{7}$.

PREDICT é um escore para pacientes com síndromes coronarianas agudas que utiliza informações clínica com a finalidade de estratificar o risco de mortalidade em 30 dias, 2 e 6 anos, independentemente do sexo e do tipo de doença coronariana ${ }^{4}$.

Uma estratificação de risco para ser empregada deve possuir variáveis simples, de rápida coleta, fácil memorização, sem a dependência de exames complementares e/ou invasivos e deve apresentar cálculos fáceis $^{3}$. Essas qualidades são encontradas no TIMI RISK SCORE (TR), o qual foi utilizado para infarto agudo do miocárdio com supradesnivelamento do segmento ST (IAM), sendo descrito originalmente por Morrow et al. ${ }^{8}$, baseado em dados clínicos de 15.060 pacientes (Tabela 1), rotineiramente colhidos na admissão ao hospital. Esse escore de risco visa avaliar o prognóstico e a evolução desses pacientes quanto ao óbito em um período de 30 dias.

Tabela 1. Tabela de estratificação de riscos para IAM com supra de ST

\begin{tabular}{ll}
\hline Critérios & Pontuação \\
\hline Idade $^{3} \quad$ 75 anos & 3 \\
65-74 anos & 2 \\
Diabetes ou HAS ou Angina & 1 \\
PA < 100 mmHg & 3 \\
FC > 100 bpm & 2 \\
Killip II-IV & 2 \\
Peso < 67 Kg & 1 \\
Infarto Anterior ou BRE & 1 \\
Tempo de inicio do tratamento $>4 \mathrm{~h}$ & 1 \\
\hline
\end{tabular}

\section{OBJETIVO}

Avaliar a relação da estratificação do TIMI RISK (TR), classicamente utilizado como preditor de fase aguda, em pacientes internados em Hospital Escola diagnosticados com IAM com supradesnivelamento do segmento ST após evolução de seis meses.

\section{CASUÍSTICA E MÉTODO}

Foram avaliados 97 pacientes segundo os critérios de inclusão (Tabela 2) no período entre fevereiro de 2000 e outubro de 2001, internados em Hospital Escola. A idade média dos pacientes foi de 57,6 anos, tendo o mais jovem 43 e o mais idoso 83 anos. Ocorreu predominância do sexo masculino com 57 homens e 40 mulheres.

Todos os pacientes foram estratificados segundo os dados de entrada pelo escore do TR (Tabela 1). Os critérios clínicos do TR foram avaliados com a concordância de pelo menos dois cardiologistas, o mesmo ocorreu para avaliação eletrocardiográfica, na qual utilizou-se o aparelho da marca Dixtal modelo E-P3.

Tabela 2. Critérios de inclusão no estudo

Critérios de inclusão

1) Pacientes com IAM com supradesnivelamento de segmento ST

2) Maiores de 35 anos

3) Concordância em autorizar a utilização dos dados para o estudo

4) Não estar incluso em nenhum outro protocolo de estudo

Continuaram no estudo 89 pacientes, o que corresponde a $91 \%$ do total de pacientes inicialmente avaliados. Os oito pacientes que não foram incluídos se recusaram a participar do estudo, ou não foram localizados pelo serviço de assistência social do hospital para seguimento.

O acompanhamento ambulatorial foi realizado durante 6 meses após a alta (Protocolo anexo), na disciplina de Cardiologia da Faculdade de Medicina, para avaliação dos seguintes parâmetros:

- óbito;

- necessidade de revascularização miocárdica;

- reinternação por evento cardiovascular. 


\section{Modelo do Protocolo}

\section{PROTOCOLO - Timi Risk Score}

\section{DADOS PESSOAIS}

$\mathrm{RH}$

NOME:

IDADE: SEXO: $\mathrm{M} \bullet-\mathrm{F} \bullet$

ENDEREÇO

BAIRRO:

TEL:

DATA DO IAM

TIMI RISK SCORE (MORROW, 2000)

\begin{tabular}{|c|c|}
\hline & Pontos \\
\hline & - 3 \\
\hline $\begin{array}{l}375 \\
65-74\end{array}$ & • \\
\hline DM ou HAS ou ANGINA & $\bullet$ \\
\hline $\mathrm{PA}<100 \mathrm{mmHg}$ & $\bullet$ \\
\hline $\mathrm{FC}>100 \mathrm{bpm}$ & - \\
\hline KILLIP II-IV & $\bullet$ \\
\hline PESO $<67 \mathrm{~kg}$ & • \\
\hline INFARTO ANT ou BRE & • \\
\hline TEMPO DE DOR > 4h & - \\
\hline
\end{tabular}

DATA DA INTERNAÇÃO

HOUVE ÓBITO?

SIM $\quad \bullet \quad$ DATA

NÃO

CEP:

HOUVE REVASCULARIZAÇÃO ?

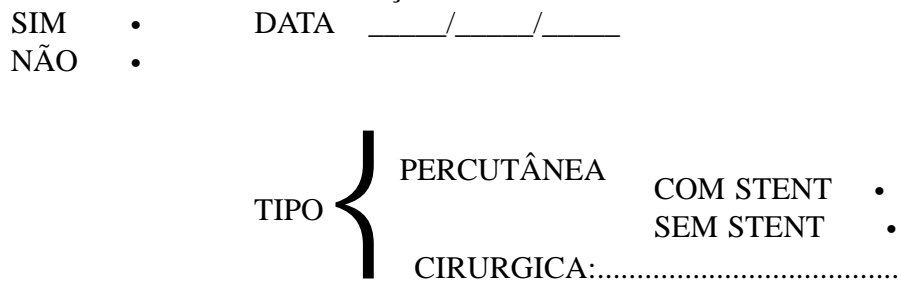

HOUVE REINTERNAÇÃO?

NIM

DATA

MOTIVO:

FAZ ACOMPANHAMENTO?

SIM •

NÃO

FAZIA USO DE ALGUM MEDICAMENTO ANTES DO IAM?

SIM • QUAL(IS)?

NÃO

REGULAR?

SIM •

NÃO • 
Rev Med (São Paulo) 2003 jan.-dez.;82(1-4):58-66.

Modelo do Protocolo

PROTOCOLO - Timi Risk Score (continuação)

FAZ USO DE ALGUM MEDICAMENTO?

SIM $\quad$ QUAL(IS)?

NÃO

REGULAR?

SIM •

NÃO •

\section{SINAIS E SINTOMAS}

\section{ICC}

Dispnéia paroxística noturna/ ortopnéia Ingurgitamento jugular

Estertores pulmonares (bases)

Cardiomegalia

Edema pulmonar agudo

Ritmo de galope ( $3^{\mathrm{a}}$ bulha)

Refluxo hepatojugular

Edema de tornozelo

Tosse noturna

Dispnéia aos esforços

Hepatomegalia

Derrame Pleural

Taquicardia (FC > $120 \mathrm{bpm})$

PRECORDIALGIA?

SIM

NÃO

EXAMES:

TESTE ERGOMÉTRICO:

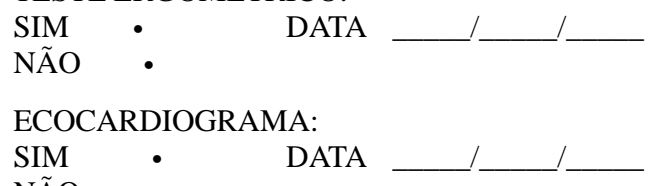

\begin{tabular}{cc} 
SIM & NÃO \\
$\bullet$ & $\bullet$ \\
$\bullet$ & $\bullet$ \\
$\bullet$ & $\bullet$ \\
$\bullet$ & $\bullet$ \\
$\bullet$ & $\bullet$ \\
$\bullet$ & $\bullet$ \\
$\bullet$ & $\bullet$ \\
$\bullet$ & $\bullet$ \\
$\bullet$ & $\bullet$ \\
\hline & $\bullet$
\end{tabular}

SIM • DATA

NÃO

CINTILOGRAFIA MIBI:
NIMO
DATA
CATE:
$\begin{array}{lll}\mathrm{SIM} & \bullet & \text { DATA } \\ \mathrm{N} \tilde{O} \mathrm{O}\end{array}$
ECG:
SIM • DATA
NÃO

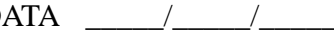

EXAME DE SANGUE:

$\begin{array}{llll}\mathrm{SIM} & \bullet & \text { DATA } & \end{array}$

OBSERVAÇÕES: 
Os pacientes foram divididos em grupos de acordo com o escore do TR: grupo I) 0-2 pontos; grupo II) 3-5 pontos; grupo III) mais de 5 pontos, correspondendo a: baixo, médio e alto risco, respectivamente (Tabela 3).

Tabela 3. Divisão dos grupos de acordo com escore

\begin{tabular}{lll}
\hline Grupos & Escore de TR & Risco \\
\hline I & 0 a 2 & Baixo \\
II & 3 a 5 & Médio \\
III & Mais de 5 & Alto \\
\hline
\end{tabular}

Os grupos foram submetidos à análise estatística pelo método de Qui-Quadrado.

\section{RESULTADOS}

Nosso estudo avaliou 89 pacientes internados em Hospital Escola com diagnóstico de IAM com supradesnivelamento do segmento ST, segundo os critérios do TR, para evolução tardia (seis meses), obtendo os resultados demonstrados na Tabela 4 .

Em seis meses de acompanhamento ambulatorial, o grupo I) apresentou $4,1 \%$ de óbitos, valor que é significantemente menor que o do grupo III) que apresentou 52\% ( $\mathrm{P}=0,05)$ (Tabela 5).

Ocorreu ainda uma tendência a um maior número de revascularização e reinternação observado entre os pacientes do grupo de maior risco em relação aos grupos de menor risco.

Tabela 4. Resultados de óbito, revascularização e reinternação nos grupos de baixo, médio e alto risco

\begin{tabular}{cccc}
\hline Grupos & Óbito & Revascularização & Reinternação \\
\hline I & $4,1 \%$ & $20,8 \%$ & $25,0 \%$ \\
II & $10,0 \%$ & $30,0 \%$ & $35,0 \%$ \\
III & $52,0 \%$ & $48,0 \%$ & $60,0 \%$ \\
\hline
\end{tabular}

Tabela 5. Análise do óbito em 6 meses através do método estatístico qui-quadrado, para um $P=0,05$

\begin{tabular}{l|cc|c|cc}
\hline \multirow{2}{*}{ Grupos } & \multicolumn{2}{|c|}{ Óbito } & \multirow{2}{*}{ Total } & \multicolumn{2}{c}{ Proporção de óbito } \\
\cline { 2 - 3 } & Sim & Não & & 0,0417 & $4,17 \%$ \\
\hline I & 1 & 23 & 24 & 0,1000 & $10,00 \%$ \\
II & 4 & 36 & 40 & 0,5200 & $52,00 \%$ \\
III & 13 & 12 & 25 & 0,2022 & $20,22 \%$ \\
& 18 & 71 & 89 & \\
\hline
\end{tabular}

\section{DISCUSSÃO}

Sendo o IAM a maior causa de óbitos na sociedade contemporânea $^{1}$, diversos autores buscam maneiras de avaliar os pacientes segundo o grau de risco (Tabela 6), de modo a direcioná-los à conduta terapêutica mais adequada, diminuindo os custos e evitando internações desnecessárias aos pacientes.

O escore de TR para infarto agudo do miocárdio com supradesnivelamento do segmento ST foi descrito originalmente por Morrow et al. ${ }^{8}$, baseado em oito variáveis de fácil pesquisa que podem ser agrupadas em uma simples tabela (Tabela 1), o que viabiliza a utilização dessa estratificação por qualquer profissional, sem a necessidade da utilização de máquinas calculadoras ou treinamento para tal função.

Os critérios utilizados no TR foram baseados em sua relativa contribuição individual para prognóstico dos pacientes com IAM. Dez variáveis foram selecionadas para formar a estratificação, sendo elas: idade, Classificação de Killip e Kimbal ${ }^{5}$, freqüência cardíaca, pressão sistólica, peso, local de acometimento cardíaco, tempo para início do tratamento, diabetes, história familiar e angina, sendo as três últimas agrupadas em um único item. 
Rev Med (São Paulo) 2003 jan.-dez.;82(1-4):58-66.

Tabela 6. Autores que avaliaram critérios de risco para óbito, revascularização e reinternação

\begin{tabular}{|c|c|c|c|}
\hline Autor & Ano & $\mathrm{N}^{\circ}$ de pacientes & Objetivo \\
\hline Moss et al. & 1974 & 518 & Preditor de óbito em 4 meses \\
\hline Moss et al. & 1978 & 866 & Caracteres que influênciam na mortalidade em 22 meses. \\
\hline Sanz et al. & 1979 & 259 & $\begin{array}{l}\text { Acompanhar a longo prazo ( } 34 \text { meses), verificando as variáveis de risco mais } \\
\text { importantes para os diferentes tipos de pacientes que sobreviveram ao IAM. }\end{array}$ \\
\hline Kornowski et al. & 1981 & 3.695 & $\begin{array}{l}\text { Relacionar reinfarto durante } 1 \text { ano e dados clínicos referentes ao primeiro } \\
\text { infarto, admissão atual, e fatores de risco. }\end{array}$ \\
\hline Normand et al. & 1987 & 14.581 & Preditor de óbito em 30 dias, para qualificar o tratamento de pacientes com IAM. \\
\hline Hillis et al. & 1990 & 3.339 & Estratificar o risco de óbito em 6 semanas. \\
\hline Jacobs et al. & 1990 & 6.134 & Estratificar o risco de mortalidade em 30 dias, 2 e 6 anos. \\
\hline Rouleau et al. & 1992 & 3.178 & $\begin{array}{l}\text { Avaliar a mortalidade de pacientes após IAM durante a internação e após } \\
1 \text { ano de alta hospitalar, assim como a recorrência de infarto e a necessidade de } \\
\text { revascularização }\end{array}$ \\
\hline Volpi et al.. & 1993 & 10.219 & Analisar o risco de óbito pós infarto em 6 meses. \\
\hline Normand et al. & 1994 & 287.469 & Relacionar comorbidades e óbito em 2 anos. \\
\hline Kerry et al. & 1995 & 41.021 & Preditor de óbito em 30 dias com utilidade na rotina clínica hospitalar. \\
\hline Morrow et al. & 2000 & 15.060 & Preditor de óbito em 30 dias com variáveis simples. \\
\hline
\end{tabular}

As variáveis utilizadas no TR são responsáveis por $97 \%$ das informações prognósticas, quando comparadas ao estudo completo das multivariáveis estudadas (GUSTO - I), os outros 3\% seriam dados pelos seguintes fatores: tabagismo, outro episódio prévio de IAM, doença vascular periférica, uso de drogas antiarrítmicas, tratamento medicamentoso para dislipidemia e sexo feminino, porém, esses foram retirados do TR de maneira a tornar sua utilização mais rápida e simples ${ }^{8}$.

O TR tem demonstrado aplicabilidade em populações distintas, mantendo sua precisão, em virtude da angina, história familiar e diabetes, que estão englobados em um único item, os quais são potencialmente diferentes conforme os hábitos e a cultura dos países.

De acordo com a literatura, a incidência de IAM no sexo masculino vem diminuindo em relação ao feminino, embora ainda seja predominante segundo vários autores. Esses dados condizem com os encontrados nesse estudo, em que 57 casos de IAM ocorreram no sexo masculino ${ }^{14}$ (Gráfico 1).
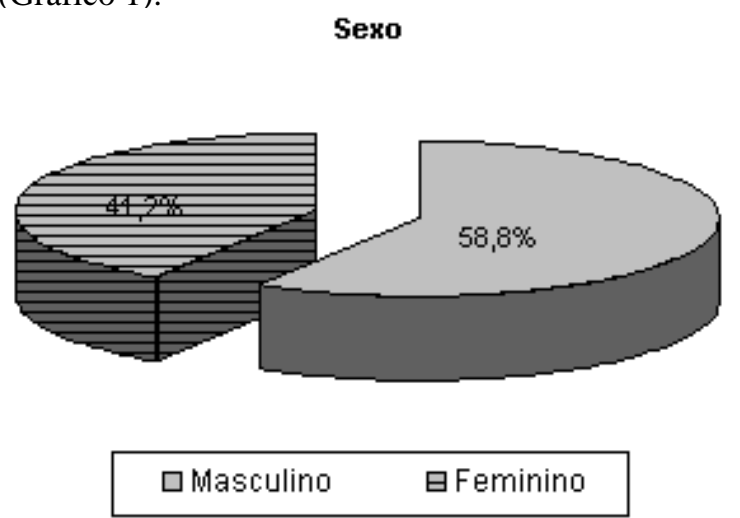

Gráfico 1. Mostrando a relação entre o número de homens e mulheres
Em relação aos critérios de inclusão todos os pacientes obrigatoriamente necessitavam ter IAM com supradesnivelamento do segmento ST, idade acima de 35 anos, visando dessa forma excluir os infartos em pacientes jovens, com possíveis quadros de doenças relacionadas a microvasculatura ou vasoespasmo sem lesão aterosclerótica, além disso não estarem participando de outros estudos, facilitando a adesão ao protocolo do presente estudo.

Alguns autores como Sanz ${ }^{18}$, Kornowski ${ }^{6}$, Normand ${ }^{13} \mathrm{e}$ Volpi ${ }^{19}$ denotaram a importância de fatores como a idade elevada e a disfunção do ventrículo esquerdo (Classificação de Killip-Kimbal $^{5}$ ), na análise prognóstica pós IAM, assumindo grande importância no TR, já que correspondem a mais de um terço de sua pontuação máxima.

Em comparação a outros modelos desenvolvidos anteriormente como o Global Utilization of Streptokinase and Tissue Plasminogen acivator for Occluded Arteries (GUSTO-I) trial de Lee et al. ${ }^{7}$, que também estratificou o risco de óbito para pacientes com IAM num período de 30 dias, o TR mostrou uma capacidade de prognóstico com eficácia quase similar, mas com uma aplicabilidade muito superior na rotina hospitalar, já que para se chegar ao valor final de pontos no GUSTO-I, estabelecendo o risco, é necessário o uso de computadores e profissionais familiarizados com tais cálculos, isso devido a esse modelo não utilizar relações lineares entre as variáveis e o risco de óbito.

Algumas análises de risco como o TR foram elaboradas para o ato da admissão hospitalar, pois utilizam somente dados clínicos facilmente verificados ${ }^{3,7,17}$, característica esta que as qualificam entre outros preditores, os quais necessitam de alguns critérios disponíveis apenas na internação $0^{4,6,10,11,12,13,19}$, postergando 
assim um encaminhamento precoce para um tratamento adequado.

Autores como Moss ${ }^{11}$, Normand ${ }^{12}, \mathrm{Hillis}^{3}, \mathrm{Lee}^{7} \mathrm{e}$ Morrow $^{8}$, criaram preditores de riscos pós IAM de curto prazo, e outros, como o de Moss ${ }^{10}$, Sanz $^{18}$, Kornowiski ${ }^{6}$, Volpi ${ }^{19}$ e Normand ${ }^{13}$, visaram um prognóstico mais prolongado. Há estudos que utilizam os mesmos fatores, porém avaliam o risco de óbito em curto e longo prazo, como o PREDICT, que analisou o risco em 30 dias, 2 e 6 anos, verificando que a taxa de mortalidade foi aumentando conforme seu escore e tempo, apresentando curvas de mesmo padrão para diferentes períodos (Gráfico 2). Igualmente, nosso estudo analisou a aplicabilidade do escore descrito originalmente por Morrow $^{8}$ para 30 dias em 6 meses.

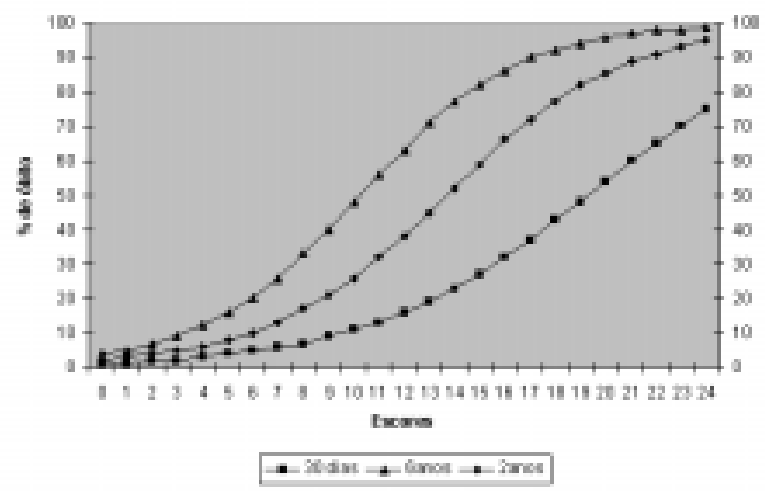

Gráfico 2. Taxa de mortalidade em 30 dias, 2 e 6 anos

Para ilustrar a utilidade e a aplicabilidade do TR, foi realizada uma análise regional no período de julho de 1997 à novembro de 1998, no qual 15.060 pacientes tratados com fibrinolíticos foram estratificados segundo os critérios do TR para o cálculo de risco de óbito em um período de 30 dias. Esse subestudo, denominado $A n$ Intravenous nPA for Treatment of Infarcting Myocardium
Early II (In TIME II), mostrou uma forte relação entre os casos de óbito e o escore de risco (Gráfico 3).

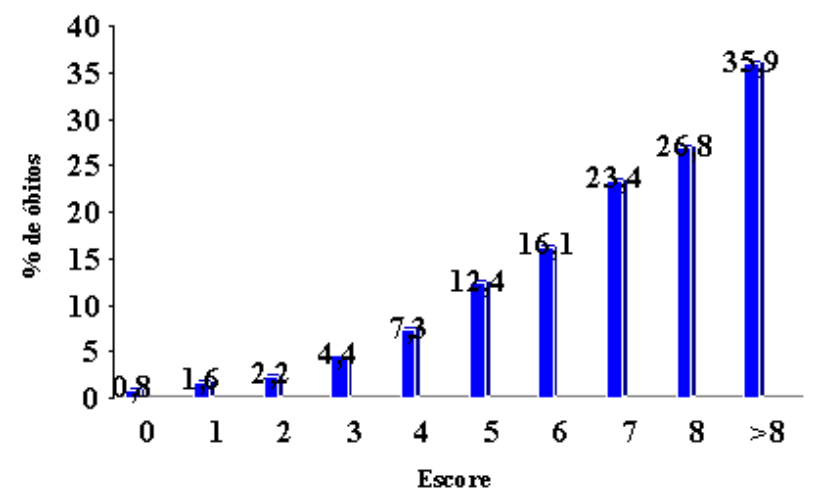

Gráfico 3. Relação entre óbito e escore de risco

No estudo realizado em Hospital Escola, utilizando 89 pacientes internados com diagnósticos de IAM, no período entre fevereiro de 2000 e outubro de 2001 e acompanhados ambulatorialmente, o TR foi utilizado como preditor de longo prazo (seis meses) para risco de óbito, revascularização e reinternação. Assim como no In TIME II, ocorreu um aumento significativo do número de eventos nos grupos de maior risco em relação aos de menor risco. Resultados que podem sugerir a aplicabilidade desse escore para longo prazo.

\section{CONCLUSÃo}

O escore de TR manteve significativo aumento de eventos nos grupos II e III em relação ao grupo I, mostrando a possível aplicabilidade do escore também como preditor de longo prazo (6 meses).

Ketzer BM, Gil E, Rodrigues GR, Kawata MF, Gun C, Luca FA. The Timi Risk score analysis in st-elevation myocardial infarction patients after 6 months. Rev Med (São Paulo) 2003 jan.-dez.;82(1-4):58-66.

\begin{abstract}
Background: The TIMI RISK (TR) score for ST-Evaluation Myocardial Infarction (STEMI) was originally describes by Morrow et al. (2000), basead on 8 variables. It was used in the In TIME study as 30-day mortality predictor and several studies successfully relate TR as prognosis index for STEMI, although few reports evaluate lete evolution of these patients and the relation whith the score. Objective: Evaluating the evolution of the STEMI interned patients in University-Based Hospitals after 6 months and the relation with the TR stratification. Methods and Results: 89 patients were clinically followed up in University-Based Hospital for 6 months after the initial determination of the TR score realized during hospital admission. The following parameters were evaluated: death, readmission for cardiovascular event, and revascularization necessity. They were classified in 3 groups according the score: Group I $(0,1,2)$; Group II $(3,4,5)$ and Group III $(>5)$, were submit to statistic analysis by chisquare test. The results of death, readmission in coronary-care unit and revascularization were respectively: GI: 4,1\%, 25,0\%, 20,8\%; GII: 10,0\%, 35,0\%, 30,0\%; GIII: 52,0\%, 60,0\%, 48,0\%. Conclusion: TR score maintained significant events increase on Groups II and III comparing to Group I; presenting the possible score applicability also at long term as predictor (6 moths).
\end{abstract}

KEYWORDS: Myocardial infarction/mortality. Follow-up studies. Myocardial revascularization. Risk factors. Inpatients. Length of stay. Chi-square. 


\section{REFERÊNCIAS}

1. Braunwald E, Gersh BJ, Rutherford JD. Chronic coronary artery disease. In: Braunwald E. Heart disease: a textbook of cardiovascular medicine. 5th ed. Philadelphia: WB Saundres; 1997. p.1265-79.

2. Goodman SG, Langer A, Ross AM, Wildermann NM. NonQ-wave versus Q-wave myocardial infarction after thrombolytic therapy: angiographic and prognostic insights from the global utilization of streptokinase and tissue plasminogem activator for occluded coronary arteries - I Angiographic substudy. Circulation 1998;97:444-50.

3. Hillis LD, Forman S, Braunwald E. Risk stratification before thrombolytic therapy in patients with acute myocardial infarction. J Am Coll Cardiol 1990;16:313-5.

4. Jacobs DA, Kroenke C, Crow R, Deshpande M, Gu DF, Gatewood L, et al. PREDICT: a simple risk score for .clinical severity and long-term prognosis after hospitalization for acute myocardial infarction or unstable angina. Circulation 1999;100:599-607.

5. Killip T, Kimball JT. Treatment of myocardial infarction in a coronary care unit - a two year experience with 250 patients. Am J Cardiol 1967;20:457-64.

6. Kornowski R, Goldbourt U, Zion M, Mandelzweig L, Kaplinsky E, et al. Predictors and long-term prognostic significnce of recurrent infarction in the year after a first myocardial infarction. Am J Cardiol 1993;72:883-8.

7. Lee KL, Woodlief LH, Topol EJ, Weaver D, Betriu A, Col $\mathrm{J}$, et al. Predictor of 30 day mortality in the era of reperfusion for acute myocardial infarction. Circulation 1995;91:165968.

8. Morrow DA, Antman EM, Charlesworth A, Cairns R, Murphy AS, Lemos JÁ, et al. Time risk score for ST elevation myocardial infartaction: a convenient, bedside, clinical score for risk assessment at presentation. Circulation 2000;102:2031-7.

9. Morrow DA, Antman EM, Giugliano RP, Cairns R, Charlesworth A, Murphy AS, et al. A simple risk index for rapid initial triage of patients with st-elevation myocardial infarction: na in time II substudy. Lancet 2001;358:1571-5.

10. Moss AJ, Bigger T, Case RB, Gillespie JA, Goldstein RE, Greenberg HM, et al. Risk stratification and survival after myocardial infarction - The Multicenter Postinfarction Research Group. N Engl J Med 1983;309:331-6.

11. Moss AJ, DeCamilla J, Henry BS, Bayer L. The early posthopital phase of myocardial infarction. Circulation 1976;54:58-64.

12. Normand SL, Glickman ME, Sharma RGVRK, McNeil BJ. Using Admission characteristics to predict short - term mortality from myocardial infarction in elderly patients. JAMA 1996;275:1322-8.

13. Normand SL, Morris CM, Fung KS, Mcneil BJ, Epstein AM. Development and validation of a claims based index for adjusting for risk of mortality: the case of acute myocardial infarction. J Clin Epidemiol 1995;48:229-43.

14. O'Hara D, McDonald I. Trends in hospital mortality following acute myocardial infarction in Victoria. NZ J Med 1997;27:431-6.

15. Porto AL, Alves MC. Angina instável. In: Porto CC. Doenças do coração. Rio de Janeiro: Guanabara Koogan; 1998. p.608-14.

16. Pozen MW, D'Agostino RB, Selker HP, Sytkowski PA, Hood WB. A predicte instrument to improve coronary care unit admission practices in acute ischemic heart disease. $\mathrm{N}$ Engl J Med 1984;310:1273-8.

17. Rouleau JL, Talajic M, Sussex B, Potvin L, Warnica W, Davies RF, et al. Myocardial infarction patients in the 1990s - Their risk factors, stratification and survival in Canada. The Canadian Assessment of Myocardial Infarction (CAMI) Study. J Am Coll Cardiol 1996;27:1119-27.

18. Sanz G, Castañer A, Betriu A, Magriña J, Roig E, Coll S, et al. Determinants of prognosis in survivors of myocardial infarction - A prospective clinical angiographic study. $\mathrm{N}$ Engl J Med 1982;306:1065-70.

19. Volpi A, Vita CD, Franzosi MG, Geraci E, Maggioni AP, Mauri F, et al. Determinants of 6-month mortality in survivors of myocardial infarction after thrombolysis - results of the GISSI-2 data base. Circulation 1993;88:416-29. 